\title{
A smallest irreducible lattice in the product of trees
}

\author{
DAVID JANZEN \\ DANIEL T WISE
}

\begin{abstract}
We produce a nonpositively curved square complex $X$ containing exactly four squares. Its universal cover $\tilde{X} \cong T_{4} \times T_{4}$ is isomorphic to the product of two 4-valent trees. The group $\pi_{1} X$ is a lattice in $\operatorname{Aut}(\tilde{X})$ but $\pi_{1} X$ is not virtually a nontrivial product of free groups. There is no such example with fewer than four squares. The main ingredient in our analysis is that $\tilde{X}$ contains an "anti-torus" which is a certain aperiodically tiled plane.
\end{abstract}

$20 \mathrm{~F} 67$

Definition 1 (Complete square complex) A square complex $X$ is a 2-complex whose $2-$ cells are squares in the sense that their attaching maps are combinatorial paths of length 4. The link of a vertex $v \in X^{0}$ is a graph whose vertices correspond to ends of 1-cells incident with $v$ and whose edges correspond to corners of 2-cells incident with $v$. Thus $\operatorname{Link}(v)$ corresponds to an $\epsilon-$ sphere about $v$ in $X$. A square complex is nonpositively curved if no link contains an embedded cycle of length $\leq 3$. A complete bipartite graph $K(m, n)$ is a graph whose vertices can be partitioned into disjoint nonempty sets $A$ and $B$ where $|A|=m$ and $|B|=n$ and such that $(a, b)$ is an edge of $K(m, n)$ if and only if $a \in A$ and $b \in B$. A square complex is a complete square complex (CSC), if $\operatorname{Link}(v)$ is a complete bipartite graph for each $v \in X^{0}$.

Complete square complexes are nonpositively curved because simplicial bipartite graphs contain no cycles of length $<4$. The direct product of two graphs is a CSC and more generally, any complex with a cover isomorphic to a product of two graphs is a CSC. A square complex is a CSC if and only if its universal cover is a direct product of two trees [9, Theorem 3.8]. In [9], the second author showed how to produce a CSC that does not have finite covers isomorphic to the product of two graphs. The CSC produced there was then used to construct examples of compact nonpositively curved square complexes whose fundamental groups are not residually finite in [9] and to construct nonpositively curved square complexes containing aperiodic flats not approximable by periodic flats in [7]. The fundamental ingredient in these examples of CSCs is the existence of an "anti-torus" (see Definition 13). Such an anti-torus was also used by Hsu and Wise [2] to construct an example of a CSC whose fundamental group has infinitely many 
isomorphism types of fixed subgroups of automorphisms. The relationship between the arithmetic of quaternions and anti-tori in certain $\operatorname{PGL}\left(2, \mathbb{Q}_{p}\right) \times \operatorname{PGL}\left(2, \mathbb{Q}_{q}\right)$ lattices was explored by Rattaggi $[5 ; 6]$. For instance, Rattaggi proves that anti-tori in irreducible lattices he studies correspond with noncommuting quaternions. A deep investigation of lattices in products of trees was conducted by Burger and Mozes [1], where the authors construct infinite simple lattices that are finitely presented and torsion-free.

Graphs of spaces provide a natural but limited source of examples of nonpositively curved square complexes. A graph of spaces $Y$ is obtained from an underlying graph $\Gamma_{Y}$ by associating a vertex space $Y_{v}$ to each vertex and edge space $Y_{e}$ to each edge of $\Gamma_{Y}$. For each edge $e$ of $\Gamma_{Y}$ with initial and terminal vertices $\iota(e)$ and $\tau(e)$, there are attaching maps $\rho_{-e}: Y_{e} \rightarrow Y_{\iota(e)}$ and $\rho_{+e}: Y_{e} \rightarrow Y_{\tau(e)}$. One constructs the space $Y$ by attaching edge spaces to adjacent vertex spaces using these attaching maps. More precisely, we first form the disjoint union $\left\{Y_{v}, Y_{e} \times[-1,1]: v \in \operatorname{Vertices}\left(\Gamma_{Y}\right), e \in\right.$ Edges $\left.\left(\Gamma_{Y}\right)\right\}$. We then form the quotient induced by $\left\{(y,+1) \sim \rho_{+e}(y): y \in Y_{e}\right\}$ and $\left\{(y,-1) \sim \rho_{-e}(y): y \in Y_{e}\right\}$. When each vertex and edge space is a graph and the attaching maps are combinatorial maps, then $Y$ is a square complex. Moreover, when the attaching maps are locally injective then $Y$ is a nonpositively curved square complex, and when the attaching maps are covering maps then $Y$ is a CSC.

We now define a square complex $X$ with only four squares that is a smallest example of a square complex whose universal cover is a direct product of two trees but which does not have a finite cover isomorphic to the product of two graphs. It follows that $\pi_{1} X$ is isometric to $F_{2} \times F_{2}$ but not commensurable with it.

Example 2 We define $X$ to be the complex obtained from the four squares in Figure 1 glued together as indicated by the labellings of their edges. Note that $X$ is a complete square complex with a unique vertex.
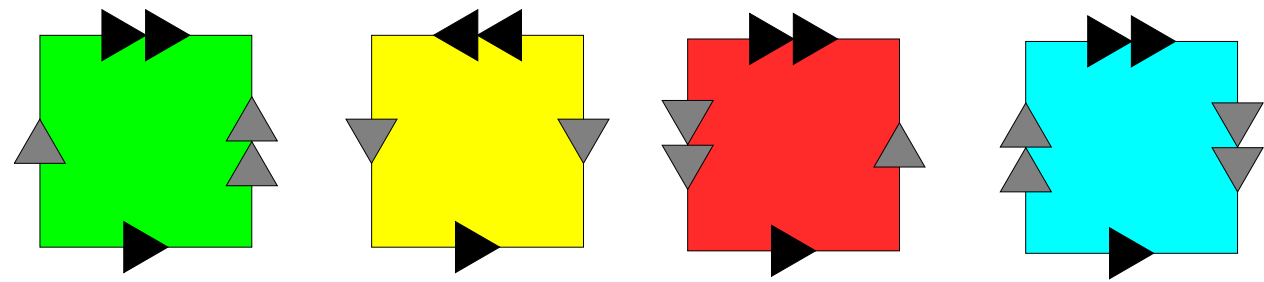

Figure 1: The four squares above are labelled to indicate how they are glued together to obtain the complex $X$. The single and double vertical edges are denoted $a$ and $b$ respectively, while the single and double horizontal edges are denoted $x$ and $y$ respectively. 
Most of the results obtained here will follow from the fact that the universal cover $\tilde{X}$ contains a certain type of aperiodic flat plane called an anti-torus. With this anti-torus as the main ingredient, we will prove the following:

Theorem 3 The action of $\pi_{1} X$ on $\tilde{X}$ is an example of an irreducible lattice in a product of trees and no smaller example exists.

We recall the following definitions:

Definition 4 Let $T_{1}, T_{2}$ be locally finite trees. A reducible lattice, $\Gamma$, in $T_{1} \times T_{2}$ is a subgroup of $\operatorname{Aut}\left(T_{1}\right) \times \operatorname{Aut}\left(T_{2}\right)$ such that $\Gamma$ is commensurable with $\Gamma_{1} \times \Gamma_{2}$, where $\Gamma_{i}$ is a lattice in $\operatorname{Aut}\left(T_{i}\right)$. If a lattice is not reducible then it is irreducible. Note that for CSCs, reducibility is equivalent to the existence of a finite cover which is a product of graphs.

Example 5 There are classical examples of irreducible lattices in semisimple Lie groups of rank $\geq 2$. For example, in the non-Archimedean case there is a beautiful construction of irreducible lattices in the product $\operatorname{PGL}\left(2, \mathbb{Q}_{p}\right) \times \operatorname{PGL}\left(2, \mathbb{Q}_{q}\right)$ for distinct primes $p, q \equiv 1(\bmod 4)$ by Mozes in [3] where many other interesting properties about these lattices are proved. Generalizations to the cases where $p, q$ are not necessarily congruent to 1 modulo 4 are found in [4]. Note that the Bruhat-Tits building associated to $\operatorname{PGL}\left(2, \mathbb{Q}_{p}\right)$ is a regular tree of degree $p+1$ on which $\operatorname{PGL}\left(2, \mathbb{Q}_{p}\right)$ acts by automorphisms.

Definition 6 A subgroup $H$ of $G$ is separable if it is the intersection of finite index subgroups of $G$. Note that $G$ is residually finite precisely when $\left\{1_{G}\right\}$ is separable.

The second author showed [8] that a compact CSC is a virtual product of graphs if and only if each of the vertex and edge groups in its virtual vertical and horizontal decompositions are separable. Since our example $X$ is not a virtual product of graphs, if we let $V=V_{X}$ denote the vertical 1-skeleton of $X$ as defined below then we reach the following conclusion:

Theorem $7 \pi_{1} V$ is not a separable subgroup of $\pi_{1} X$.

This gives a very small example of a nonseparable quasiconvex subgroup of a CAT(0) group. As an application of the above theorems, we are able to produce a CSC with only eight squares whose fundamental group is not residually finite. To do this, we "double" $X$ along $V$. 
Definition 8 Let $\bar{X}$ be an isomorphic copy of $X$ and $\bar{V}$ an isomorphic copy of $V$ inside $\bar{X}$. Then the double of $X$ along $V$ is the complex $D=(X \cup \bar{X} / V=\bar{V})$.

It is easy to verify that $D$ is itself a CSC with eight squares. Following the argument in [9], doubling a group along a nonseparable subgroup yields a group which is not residually finite. This shows:

Theorem $9 \pi_{1} D$ is not residually finite.

We do not know the answer to the following question:

Question 10 Is $\pi_{1} X$ residually finite?

Note that irreducible lattices given in Example 5 are residually finite.

\section{VH-complexes, decompositions and subdividing}

Definition 11 A square complex, $Y$, is a VH-complex if the 1-cells of $Y$ can be partitioned into two classes, $V$ and $H$, such that the attaching map of each square in $Y$ alternates between edges of $V$ and $H$. If the attaching map of each square of $Y$ preserves the orientation on the edges of $V$ (resp. $H$ ) then $Y$ is said to be vertically directed (resp. horizontally directed). We define $V_{X}=V \cup X^{0}$ to be the vertical 1-skeleton of $X$ and $H_{X}=H \cup X^{0}$ to be the horizontal 1-skeleton.

The complex $X$ is not horizontally directed because the second square from the left in Figure 1 does not preserve the horizontal orientation. We can however pass to a horizontally directed double cover of $X$. This covering space corresponds to the homomorphism $\pi_{1} X \rightarrow \mathbb{Z}_{2}$ sending the horizontal generators of $\pi_{1} X$ to 1 and the vertical generators to 0 . The eight squares of this cover, $\hat{X}$, are shown in Figure 2 .

Note that $\hat{X}$ has two 0 -cells and that it has two 1 -cells for each label. For example, in the case of the horizontal edges, for each label we have one 1-cell oriented from the black 0 -cell to the white 0 -cell and another from the white 0 -cell to the black 0 -cell. In Figure 3 we have relabeled these 1 -cells, a darker label given to the 1 -cells oriented from the white 0 -cell to the black 0 -cell and a lighter label given to the 1 -cell oriented from the black 0 -cell to the white 0 -cell. In Figure 3 we have also reoriented the 1 -skeleton of $\hat{X}$, so that we are able to see that $\widehat{X}$ is horizontally directed. The squares in the bottom row of Figure 3 have been flipped by a reflection as this helps to reveal the structure of $\hat{X}$ as a graph of spaces which we describe next. This structure will become the focal point of our analysis below. 

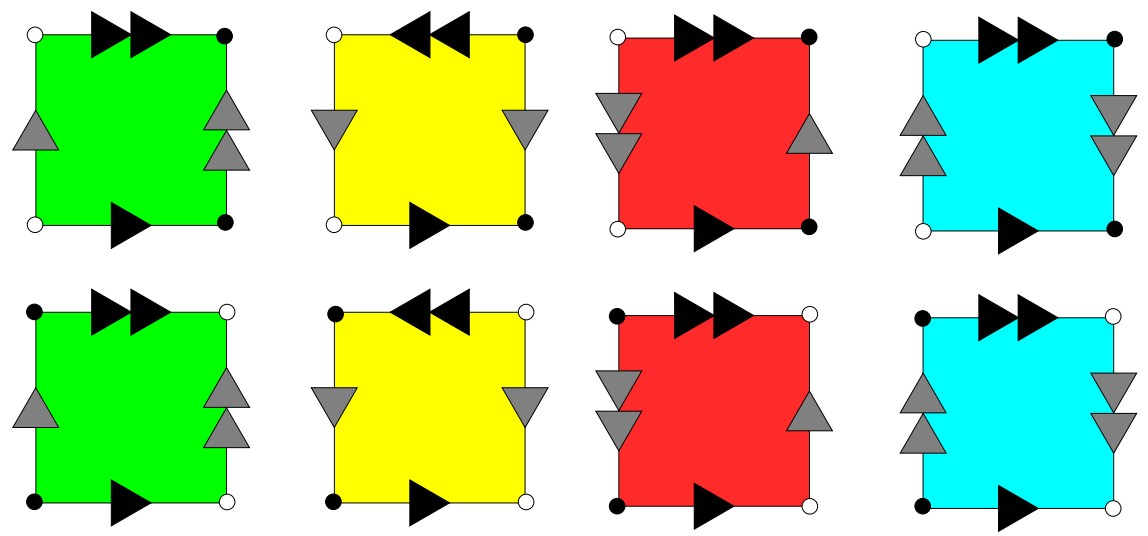

Figure 2: These eight squares are obtained as a horizontally directed double cover of the original complex $X$.
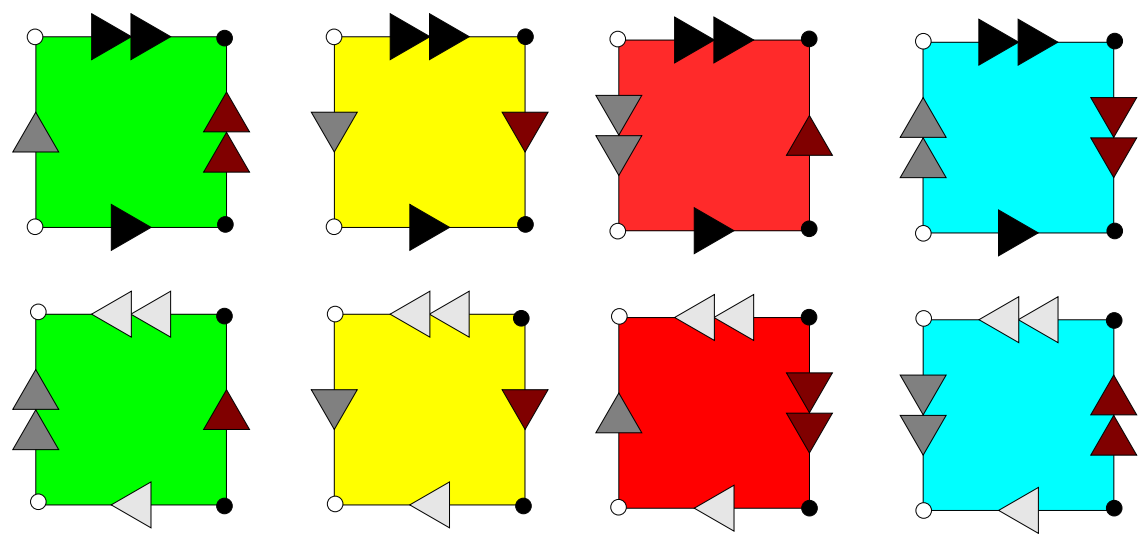

Figure 3: These eight squares reveal the structure of $\hat{X}$ as a graph of spaces.

The complement of the vertical 1 -skeleton, $V_{\hat{X}}$, of $\hat{X}$ is the cartesian product of an open 1-cell with the graph $\triangle$. Attaching this space to $V_{\hat{X}}$ on the left and right sides as indicated in Figure 4 we recover the complex $\hat{X}$. Note that the labeled edges in Figure 4 correspond to the vertical edge labels in Figure 3.

\section{The anti-torus in $X$}

Definition 12 (Periodic flat) A flat $\Lambda$ in a space $\tilde{Y}$ is an isometrically embedded copy of Euclidean space $\mathbb{E}^{n}$, for $n>1$. Let $G$ act on $Y$ by isometries. Then $\Lambda$ is periodic if $\operatorname{Stab}(\Lambda)$ contains a $\mathbb{Z}^{n}$ subgroup acting properly and cocompactly on $\Lambda$. 

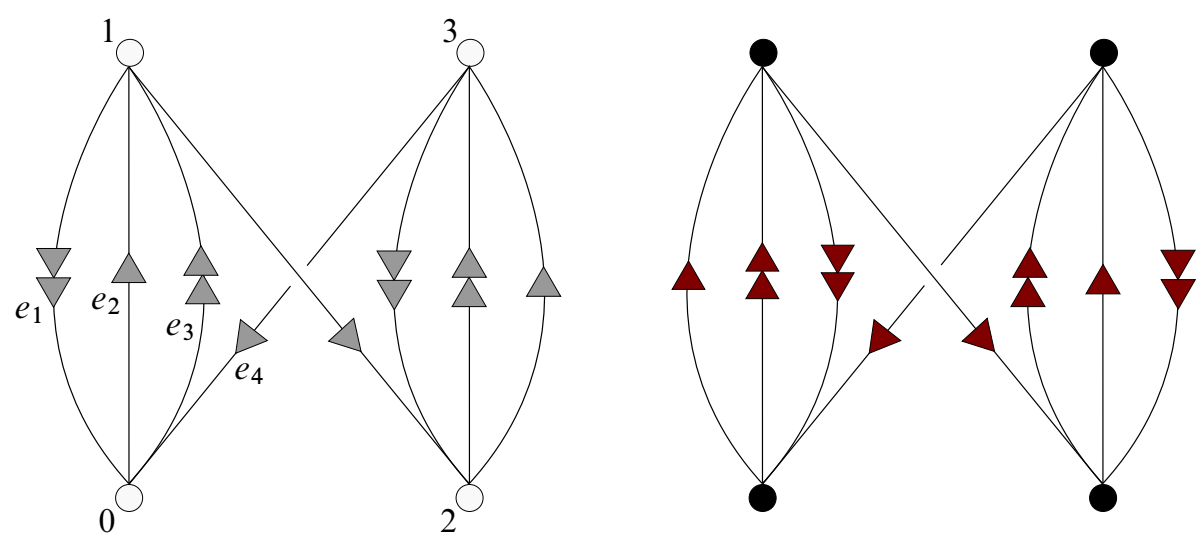

Figure 4: The graphs on the left and right, denoted $L$ and $R$ respectively, indicate the edge space structure of $\hat{X}$ as an amalgamated product.

Otherwise, $\Lambda$ is aperiodic. When $\tilde{Y}$ is the universal cover of $Y$ and $G=\pi_{1} Y$ then the above condition for a plane to be periodic is equivalent to requiring that the map $\Lambda \rightarrow Y$ factor through a torus. In other words, when $\Lambda$ is periodic, we have $\Lambda \rightarrow T \rightarrow Y$ where $T$ is a flat torus and $\Lambda \rightarrow T$ is a covering map so $T=\Lambda / \mathbb{Z}^{n}$.

As we are working with square complexes, the natural setting will be flat planes appearing in the universal cover $\tilde{Y}$ with the natural action $\pi_{1} Y \curvearrowright \tilde{Y}$.

Definition 13 (Anti-torus) Suppose $Y$ is a CSC with a VH-structure and $y \in Y^{0}$. Let $s_{h}$ and $s_{v}$ denote closed loops in $Y$ based at $y$ in the horizontal and vertical directions respectively. Then $\widetilde{s_{h}} \times \widetilde{s_{v}}$ is a planar subspace of $\tilde{Y}$. If this plane is aperiodic then $\widetilde{s_{h}} \times \widetilde{s_{v}}$ is called an anti-torus.

Our goal is now to show that the universal cover of the complex $X$, defined above, contains an anti-torus. This anti-torus is partly illustrated in Figure 5.

We begin with some notation.

For the spaces $L$ and $R$ in Figure 4, we will identify the single and double arrows with their images in $X$. Thus, the single grey arrows in $L$ and single brown arrows in $R$ are both denoted $a$, and similarly double grey and brown arrows are denoted $b$. The graph isomorphism which translates $L$ to $R$ will be denoted by $\tau: L \rightarrow R$. This isomorphism takes the upper left vertex of $L$ to the upper left vertex of $R$ and so on. Note that the white and black vertices of $L$ and $R$ in Figure 4 correspond respectively to the white vertex and black vertex of $\hat{X}$ in Figure 3. Given a path $\sigma$ in the vertical generators $a, b$ of $\pi_{1} X$, we create a new path $\sigma_{i}$ in $a, b$ by lifting $\sigma$ to a path in $L$ 


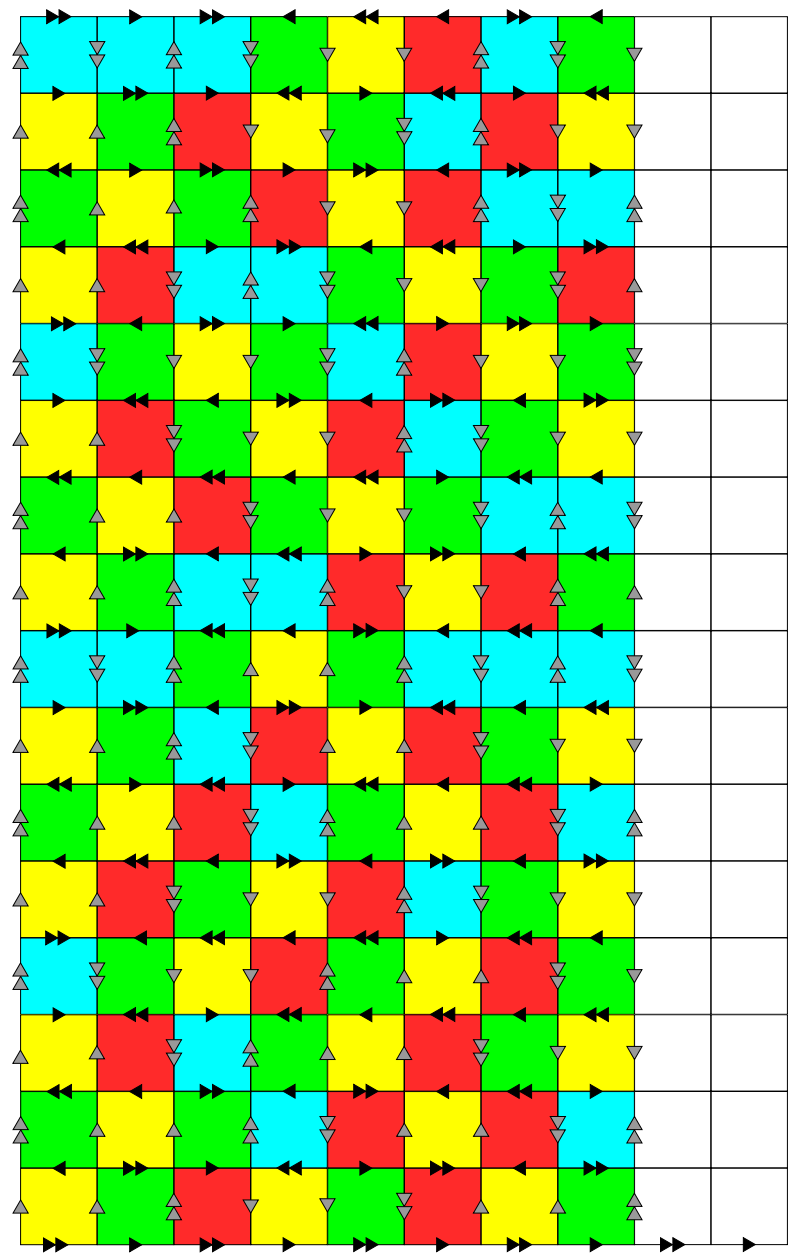

Figure 5: The convex hull of the vertical axis periodically labelled $(a b)^{\infty}$ and the horizontal axis periodically labelled $(x y)^{\infty}$ uniquely determines a plane $\Pi$ in the universal cover $\tilde{X}$. The plane is tiled by squares of $X$ as indicated by the small region of the northeast quadrant which has been tiled above. $\Pi$ is an anti-torus as described below.

based at the vertex $i$ (for some $i=0,1,2,3$ ) and translating this path to $R$ via $\tau$. We denote this composition by $\phi_{i}$, so $\sigma_{i}=\phi_{i}(\sigma)$. For example $\phi_{3}\left(a^{2} b a^{-1}\right)=a b a^{-2}$ and $\phi_{0}\left(a^{2} b a^{-1}\right)=b a^{2} b$.

There is a covering space automorphism $\psi$ of $L$ that sends the vertex $i$ to the vertex $(i+2) \bmod 4$. For the labelled edges in $L$, define $\bar{e}_{j}=\psi\left(e_{j}\right)$ for $j=1,2,3,4$. Similarly if $\sigma$ is a path in $L$, let $\bar{\sigma}=\psi(\sigma)$. If $\sigma$ is based at 0 then $\bar{\sigma}$ is based at 2 . 
Let $\pi_{1}(L, i)$ denote the fundamental group of $L$ based at the vertex $i$, for each $i=0,1,2,3$. Then $\sigma \notin \pi_{1}(L, 0)$ means that $\sigma$ is not a loop based at the vertex 0 . Let $\#_{b}(\sigma)$ be the exponent sum of $b$ 's occurring in the word $\sigma$. For example $\#_{b}\left(b^{-3} a^{2} b^{5}\right)=2$. We then have the following lemma from which we can deduce that $\tilde{X}$ contains an anti-torus.

Lemma 14 Let $\sigma$ be a path, based at 0 , in the vertical generators $a, b$ of $\pi_{1} X$ such that $\sigma$ has even length. If $\sigma \notin \pi_{1}(L, 0)$ and $\#_{b}(\sigma)$ is odd, then $\phi_{0}\left(\sigma^{2}\right) \notin \pi_{1}(L, 1)$ and $\#_{b}\left(\phi_{0}\left(\sigma^{2}\right)\right)$ is also odd.

Proof Since $\sigma$ has even length and $\sigma \notin \pi_{1}(L, 0)$, we know that $\sigma$ ends at 2. Thus, $\sigma^{2}=\sigma \cdot \bar{\sigma}$ and it follows that $\sigma^{2} \in \pi_{1}(L, 0)$. Therefore, for each $j$, the path $\sigma^{2}$ traverses $e_{j}$ exactly the same number of times as it traverses $\overline{e_{j}}$.

In particular, if the number of times $\sigma^{2}$ traverses $e_{1}$ and $\overline{e_{3}}$ are $k_{1}$ and $k_{2}$ respectively then the edges $e_{1}, e_{3}, \overline{e_{1}}, \overline{e_{3}}$ are traversed $2\left(k_{1}+k_{2}\right)$ times by $\sigma^{2}$. This means that $\#_{b}\left(\sigma^{2}\right)=2\left(k_{1}+k_{2}\right)$. But $\#_{b}\left(\sigma^{2}\right)=2 \#_{b}(\sigma)$ and $\#_{b}(\sigma)$ is odd by hypothesis. Hence $k_{1}+k_{2}$ is odd. Note that this is exactly what is required for $\phi_{0}\left(\sigma^{2}\right) \notin \pi_{1}(L, 1)$.

As $\sigma^{2} \in \pi_{1}(L, 0)$, it traverses the pair $e_{4}, \overline{e_{4}}$ an even number of times. Combined with the facts that $k_{1}+k_{2}$ is odd and that $\sigma$ has even length, this implies that the remaining edges $e_{2}, e_{3}, \overline{e_{1}}, \overline{e_{2}}$ are traversed an odd number of times which means that $\#_{b}\left(\phi_{0}\left(\sigma^{2}\right)\right)$ is odd.

Remark 15 We note that $\sigma=a^{2} \notin \pi_{1}(L, 0)$ is a word of even length, but $\phi_{0}\left(\sigma^{2}\right)=$ $b a b^{-1} a \in \pi_{1}(L, 1)$. Thus Lemma 14 fails without the added condition on the exponent sum of the $b$ 's.

Remark 16 While we have given the argument for $\sigma$ based at the vertex 0 , equivalent arguments also hold when $\sigma$ is based at any of the other vertices. We thus have the following general statement: Let $\sigma$ have even length. For any $i=0,1,2,3$ if $\sigma \notin \pi_{1}(L, i)$ and $\#_{b}(\sigma)$ is odd, then $\phi_{i}\left(\sigma^{2}\right) \notin \pi_{1}(L, i \pm 1)$ and $\#_{b}\left(\phi_{i}\left(\sigma^{2}\right)\right)$ is also odd.

We can now establish the goal of this section.

Proposition 17 The plane $\Pi$ is an anti-torus.

Proof Let $W_{k}(m)$ denote the length $k$ positive horizontal path in $\Pi$ beginning at the endpoint of the vertical $a b$ path with $m$ letters. For positive horizontal words, one shows by induction on $k$ that $W_{k}(m)=W_{k}\left(m+2^{k+1}\right)$. This implies that the first $k$ 
letters of $W_{k+1}(m)$ and $W_{k+1}\left(m+2^{k+2}\right)$ are equal so it suffices to show that their last letters are distinct. Now the vertical word connecting the endpoints of $W_{k}(m)$ and $W_{k}\left(m+2^{k+1}\right)$ is of length $2^{k+1}$ satisfying the conditions of Remark 16 . Hence by Remark 16, we have that $W_{k+1}(m) \neq W_{k+1}\left(m+2^{k+2}\right)$. Consequently, by induction, $\Pi$ cannot be periodic.

Lemma 18 For a square complex $Y$, if $\tilde{Y}$ contains an anti-torus then $Y$ is irreducible.

Proof Suppose $\hat{X}$ was a degree $d$ finite cover of $X$ isomorphic to a product of two graphs. The vertical/horizontal structure of $X$ is preserved passing to the cover $\hat{X}$, so $(a b)^{d}$ and $(x y)^{d}$ represent immersed closed paths in $\hat{X}$ with vertical and horizontal orientations respectively. This yields an immersion of the flat torus $T=(a b)^{d} \times(x y)^{d}$ into $\hat{X}$. But this determines an embedding of the periodic flat plane $\widetilde{T}$ into $\tilde{X}$. This contradicts the uniqueness of the aperiodic flat plane $\Pi$ with axes $(a b)^{\infty}$ and $(x y)^{\infty}$ as constructed earlier.

Corollary 19 The complex $X$ has no finite cover isomorphic to a product of two graphs.

We have thus shown that $\pi_{1} X$ is not commensurable with $F_{2} \times F_{2}$ even though the two groups are isometric since they have isomorphic unlabelled Cayley graphs.

The action of $\pi_{1} X$ on $\hat{X}$ gives an example of an irreducible lattice, ie, the action is discrete and cocompact and it is not virtually a product of lattices (in the horizontal and vertical directions). In the next section we will show that no smaller example exists. It seems likely however that the example $X$ is not the unique example of an irreducible lattice with four squares. Our computations suggest that the example in Figure 6 with four squares also contains an anti-torus. Note that this example does not have a consistent global $\mathrm{VH}$-orientation. If the black single and double arrows are labeled $a$ and $b$ respectively and the grey single and double arrows are labeled by $c$ and $d$, then we guess that the periodically labeled axes $(a d)^{\infty}$ and $(b c)^{\infty}$ determine an anti-torus in the example of Figure 6.

\section{No irreducibles with fewer than 4 squares}

Consider a CSC, $Y$, with at least one square and at most three squares. We may suppose that $Y$ has no free faces since free faces may be collapsed yielding a smaller CSC without free faces. Indeed, if $Y$ does have free faces then the universal cover $\widetilde{Y}$ is the product of two "leafy trees". Collapsing the leaves of these trees, we obtain 

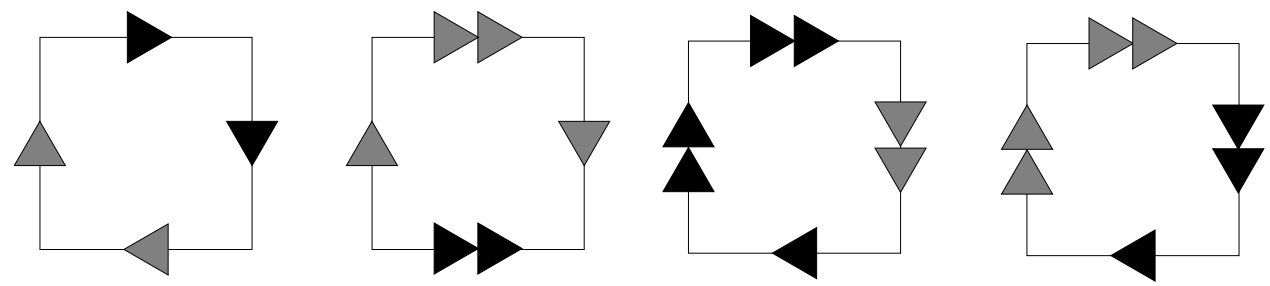

Figure 6: Another four square example which may contain an anti-torus.

a leafless core for each tree and the product of these leafless trees is stabilized under the action of $\pi_{1} Y$. Hence, collapsing the free faces of $Y$ yields a complex, $\bar{Y}$, whose universal cover is still a the product of two trees but now $\bar{Y}$ is a CSC without free faces. It is easy to see that $Y$ is a virtual product if and only if $\bar{Y}$ is.

Note that the assumption that there are no free faces is equivalent to assuming that there is no $0-$ cell, $v$, such that $\operatorname{Link}(v) \cong K(1, n)$.

By a counting argument, we prove that if $Y$ has no free faces, then the link of each 0 -cell of $Y$ is of the form $K(2, n)$ for some $n$. Suppose that $Y$ has three squares. If $Y$ has a single 0 -cell then the link of that 0 -cell is isomorphic to $K(m, n)$ where $m n=12$, since three squares have 12 corners which contribute 12 edges to the link. Each 1-cell of $Y$ contributes two vertices to the link so $m+n$ is even. Thus $K(1,12)$ and $K(3,4)$ are excluded as possibilities, and so the link is isomorphic to $K(2,6)$. Note that $K(1,12)$ is also excluded because there are no free faces. Similarly, if $Y$ has two 0 -cells $v_{1}$ and $v_{2}$, then there are two possibilities: $\operatorname{Link}\left(v_{1}\right) \cong K(2,2)$ and $\operatorname{Link}\left(v_{2}\right) \cong K(2,4)$ or $\operatorname{Link}\left(v_{1}\right) \cong K(2,3)$ and $\operatorname{Link}\left(v_{2}\right) \cong K(2,3)$. If $Y$ has three $0-$ cells $v_{1}, v_{2}$ and $v_{3}$ then we must have $\operatorname{Link}\left(v_{i}\right) \cong K(2,2)$ for each $i=1,2,3$. If $Y$ has four or more 0 -cells then at least one 0 -cell has a link of the form $K(1, n)$ which was excluded above. The cases where $Y$ has two squares or one square are analogous.

Since the link of every 0 -cell of $Y$ is isomorphic to $K(2, n)$, we have that $\tilde{Y} \cong T \times \mathbb{R}$. If this were not the case and $\tilde{Y} \cong T_{1} \times T_{2}$ with $T_{i} \not \mathbb{R}$ then for some $p_{1} \in T_{1}$ and $p_{2} \in T_{2}$ we would have valence $\left(p_{1}\right) \geq 3$ and valence $\left(p_{2}\right) \geq 3$. But then in the product $T_{1} \times T_{2}$ we would have $\operatorname{Link}\left(p_{1}, p_{2}\right) \cong K\left(\operatorname{valence}\left(p_{1}\right)\right.$, valence $\left.\left(p_{2}\right)\right)$ which is impossible.

As $\pi_{1} Y$ acts freely and properly on $\tilde{Y} \cong T \times \mathbb{R}$, we obtain that $\pi_{1} Y$ is virtually $\mathbb{Z} \times F_{n}$. Indeed, either $\tilde{Y} \cong \mathbb{R} \times \mathbb{R}$ in which case $Y$ is a Klein bottle or torus and hence easily a virtual product of graphs, or $\tilde{Y} \cong \mathbb{R} \times T$ with $T \not \mathbb{R}$ in which case $Y$ is a graph of free groups with cyclic edge groups since the action of $\pi_{1} Y$ preserves each factor. Again, $Y$ is easily a virtual product as in [9]. 
We have thus shown that every CSC with $\leq 3$ squares is virtually a product of graphs and hence every lattice is reducible. Reasoning along the same lines as above, one can also show that every connected CSC with 5 or 7 squares is a virtual product of graphs.

Acknowledgments We are grateful to the referee for the helpful corrections that improved this paper.

David Janzen was supported by an NSERC Canada Graduate Scholarship and Daniel Wise was supported by NSERC.

\section{References}

[1] M Burger, S Mozes, Lattices in product of trees, Inst. Hautes Études Sci. Publ. Math. (2000) 151-194 (2001) MR1839489

[2] T Hsu, D T Wise, Groups with infinitely many types of fixed subgroups, Israel J. Math. 144 (2004) 93-107 MR2121535

[3] S Mozes, Actions of Cartan subgroups, Israel J. Math. 90 (1995) 253-294 MR1336326

[4] D Rattaggi, Computations on groups acting on a product of trees: normal subgroup structures and quaternion lattices, PhD thesis, ETH Zürich (2004)

[5] D Rattaggi, Anti-tori in square complex groups, Geom. Dedicata 114 (2005) 189-207 MR2174099

[6] D Rattaggi, G Robertson, Abelian subgroup structure of square complex groups and arithmetic of quaternions, J. Algebra 286 (2005) 57-68 MR2124808

[7] D T Wise, A flat plane that is not the limit of periodic flat planes, Algebr. Geom. Topol. 3 (2003) 147-154 MR1997317

[8] D T Wise, Subgroup separability of the figure 8 knot group, Topology 45 (2006) 421463 MR2218750

[9] D T Wise, Complete square complexes, Comment. Math. Helv. 82 (2007) 683-724 MR2341837

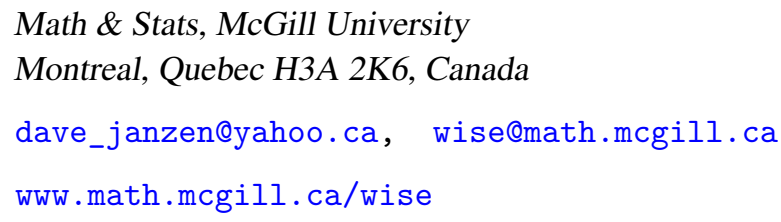

Received: 4 April 2007 Revised: 9 March 2009 